\title{
An improved SPH method for modeling liquid sloshing dynamics
}

\author{
J.R. Shao ${ }^{a}$, H.Q. Li ${ }^{b}$, G.R. Liu ${ }^{c}$, M.B. Liu ${ }^{\mathrm{a}, *}$ \\ ${ }^{a}$ LHO, Institute of Mechanics, Chinese Academy of Sciences, Beijing 100190, China \\ ${ }^{\mathrm{b}}$ School of Information and Electronics, Beijing Institute of Technology, Beijing 100081, China \\ ${ }^{\mathrm{c}}$ Aerospace Systems, University of Cincinnati, Cincinnati, $\mathrm{OH}$ 45221-0070, USA
}

\section{A R T I C L E I N F O}

\section{Article history:}

Received 13 October 2011

Accepted 20 February 2012

Available online 12 March 2012

\section{Keywords:}

Smoothed particle hydrodynamics (SPH)

Liquid sloshing

Numerical accuracy

Turbulence model

Solid boundary treatment

\begin{abstract}
A B S T R A C T
Smoothed particle hydrodynamics (SPH) is a popular meshfree, Lagrangian particle method with attractive features in modeling liquid sloshing dynamics, which is usually associated with changing and breakup of free surfaces, strong turbulence and vortex, and "violent" fluid-solid interaction. This paper presents an improved SPH method for modeling liquid sloshing dynamics. Firstly, modified schemes for approximating density (density correction) and kernel gradient (kernel gradient correction, or KGC) have been used to achieve better accuracy with smoother pressure field. Secondly, the Reynolds Averaged turbulence model is incorporated into the SPH method to describe the turbulence effects. Thirdly, a coupled dynamic solid boundary treatment (SBT) algorithm has been proposed to improve the accuracy near the solid boundary areas. The new SBT algorithm consists of a kernel-like, soft repulsive force between approaching fluid and solid particles, and a reliable numerical approximation scheme for estimating field functions of virtual solid particles. Three numerical examples are modeled using this improved SPH method, and the obtained numerical results agree well with experimental observations and results from other sources.
\end{abstract}

(c) 2012 Elsevier Ltd. All rights reserved.

\section{Introduction}

Sloshing refers to the movement of liquid inside a partiallyfilled container due to external excitations. When the amplitude of an external excitation is very large or its frequency is close to the natural frequency of the liquid sloshing system, the liquid inside the container can exhibit violent oscillations, and exert strong impact load on the container [1]. Liquid sloshing can be frequently observed in daily life and in engineering and sciences, and can be of great importance both in theory and practices. For example, large liquid sloshing in an oil or liquefied natural gas (LNG) ship can result in local breakages and global instability to the ship, and can then further lead to leakage of oil, and capsizing of ship. The movement of water in a reservoir when experiencing an earthquake can produce tremendous impact pressure on the dam of the reservoir. The sloshing of liquefied fuel inside the fuel tank in an aeronautic or astronautic craft can disturb or even breakdown normal navigation of the craft.

Due to the ever-increasing interests on liquid sloshing dynamics, many researchers have conducted theoretical, experimental and numerical simulation works in this area. Theoretical researches are usually valid for simple cases with linear or weakly

\footnotetext{
* Corresponding author. Tel.: +861082544024.

E-mail addresses: shaojiaru@imech.ac.cn (J.R. Shao), huiqili@bit.edu.cn (H.Q. Li), liugr@ucmail.uc.edu (G.R. Liu), liumoubin@imech.ac.cn (M.B. Liu).
}

nonlinear liquid sloshing dynamics. Experimental works are generally expensive and sometimes certain physical phenomena related to liquid sloshing cannot be scaled in a practical experimental setup. Recently more and more researches on liquid sloshing are focused on numerical simulations with the advancement of the computer hardware and computational techniques. A number of researchers have provided comprehensive reviews on the problem of liquid sloshing, and the related numerical simulation methods [2-4]. Most of the numerical simulations are focused on grid-based methods, such as finite difference method (FDM) [5,6], finite element method (FEM) [7,8], and boundary element method (BEM) [9-12]. As a complex fluid motion, sloshing usually involves changing and breakup of free surfaces, strong turbulence and vortex, and violent fluid-solid interaction. It is therefore difficult for traditional grid-based numerical methods to model liquid sloshing problems. For example, traditional FEM cannot treat large fluid deformation very well, and mesh adjustment or rezoning can be necessary in model liquid sloshing. Traditional FDM requires special algorithms such as volume-of-fluid (VOF) [13,14] and Level Set [15] to track changing free surfaces or moving interfaces when modeling liquid sloshing.

Recent developments in so-called meshfree and particle methods provide alternatives for traditional numerical methods in modeling free surface flows such as liquid sloshing dynamics $[16,17]$. Among the meshfree and particle methods, smoothed particle hydrodynamics (SPH) [18-20] is unique and has some obvious 
advantages. In SPH, the state of a system is represented by a set of particles, which possess individual material properties and interact with each other within a certain range defined as a support domain by a weight function or smoothing function [21]. Flow field variables (such as density, velocity, acceleration) can be obtained through approximating the governing equations which are discretized on the set of particles. The SPH method was originally invented to solve astrophysical problems in the open space such as binary stars, stellar collisions and motion near black holes. Later, it has been extensively studied and extended to different problems in engineering and sciences, such as multi-phase [22,23] and multi-scale [24] flows, high strain hydrodynamics with material strength [25-28], explosion and underwater explosion [29-31], and many others [32-34]. As a comparatively new computational method, SPH combines the advantages of meshfree, Lagrangian and particle methods. Particles are used to represent the state of a system and these particles can freely move according to internal particle interactions and external forces. Therefore it can naturally obtain history of fluid motion, and can easily track material interfaces, free surfaces and moving boundaries. The meshfree nature of SPH method remove the difficulties due to large deformations since SPH uses particles rather than mesh as a computational frame to approximate related governing equations. These features of SPH make it fairly attractive in modeling free surface and multiphase flows with violent fluid-solid interactions, especially in ocean and coastal hydrodynamics and offshore engineering. Typical applications of SPH method in this area include dam breaking, wave dynamics (waver generation, wave breaking, and wave interaction with other structures), water filling and water discharge (to and from a water tank or reservoir), and water entry.

There are a few literatures addressing the application of SPH method to liquid sloshing dynamics. For example, Iglesias et al. [35] simulated the anti-roll tanks and sloshing type problems. Rhee and Engineer studied liquid tank sloshing with Reynolds-averaged Navier-Stokes [36]. Souto-Iglesias et al. [37] assessed the liquid moment amplitude in sloshing type problems with smooth particle hydrodynamics. Anghileri et al. [38] investigated the fluid-structure interaction of water filled tanks during the impact with the ground. Delorme et al. [39] simulated the sloshing loads in LNG tankers with SPH. These works have demonstrated the feasibility of SPH method in modeling liquid sloshing dynamics. It is noted that previous works are generally based on traditional SPH method, which is believed to have poor computational accuracy. Also previous works usually did not incorporate turbulence models into $\mathrm{SPH}$ equations of motion. While when modeling turbulence and vortex in liquid sloshing, turbulence models can be very important.

In this paper, an improved SPH model for numerical simulation of liquid sloshing dynamics shall be presented. The SPH model includes an improved approximation scheme with corrections on kernel gradient and density, an enhanced solid boundary treatment algorithm, and the Reynolds Averaged Navier-Stokes (RANS) turbulence model [40]. The effectiveness of the SPH model shall be validated by three liquid sloshing problems.

\section{SPH methodology}

\subsection{SPH equations of motion}

The conventional SPH method was originally developed for hydrodynamic problems in which the governing equations are in strong form of partial differential equations of field variables such as density, velocity, and etc. There are basically two steps in obtaining an SPH formulation, kernel and particle approximations. The kernel approximation is to represent a function and its derivatives in continuous form as integral representation using the smoothing function and its derivatives. In the particle approximation, the computational domain is discretized with a set of particles. A field function and its derivative can then be written in the following forms [34]

$<f\left(\boldsymbol{x}_{i}\right)>=\sum_{j=1}^{N} \frac{m_{j}}{\rho_{j}} f\left(\boldsymbol{x}_{j}\right) W\left(\boldsymbol{x}_{i}-\boldsymbol{x}_{j}, h\right)$,

$<\nabla \cdot f\left(\boldsymbol{x}_{i}\right)>=\sum_{j=1}^{N} \frac{m_{j}}{\rho_{j}} f\left(\boldsymbol{x}_{j}\right) \nabla_{i} W_{i j}$,

where $\left\langle f\left(\boldsymbol{x}_{i}\right)>\right.$ is the approximated value of particle $i ; f\left(\boldsymbol{x}_{j}\right)$ is the value of $f(\boldsymbol{x})$ associated with particle $j ; \boldsymbol{x}_{i}$ and $\boldsymbol{x}_{j}$ are the positions of corresponding particles; $m$ and $\rho$ denote mass and density respectively; $h$ is the smooth length; $N$ is the number of the particles in the support domain; $W$ is the smoothing function representing a weighted contribution of particle $j$ to particle $i$. The smoothing function is sometimes referred to as kernel or kernel function, and it should satisfy some basic requirements, such as normalization condition, compact supportness, and Delta function behavior. This conditions are needed to ensure convergence and reproducibility of function approximation A detailed discussion on the smoothing function, its basic requirements and constructing conditions can found in $[20,21]$. In this paper, the frequently used cubic spline is employed for general purposes.

For incompressible viscous hydrodynamic problems, the Lagrangian form governing Navier-Stokes $(\mathrm{N}-\mathrm{S})$ equation can be written as

$\frac{\mathrm{d} \rho}{\mathrm{d} t}=-\rho \nabla \cdot \boldsymbol{v}$,

$\frac{\mathrm{d} \boldsymbol{v}}{\mathrm{d} t}=-\frac{1}{\rho} \nabla p+\frac{\mu}{\rho} \nabla^{2} \boldsymbol{v}+\mathbf{g}$

where, $v, p, g$ and $\mu$ denote velocity vector, pressure, gravity and dynamic viscosity respectively. Substituting the SPH approximations for a function and its derivative to $\mathrm{N}-\mathrm{S}$ equations, after some trivial transformation, the SPH equations of motion for $\mathrm{N}-\mathrm{S}$ equations can be obtained as

$$
\begin{aligned}
& \frac{\mathrm{d} \rho_{i}}{\mathrm{~d} t}=\sum_{j=1}^{N} m_{j} v_{i j} \nabla_{i} W_{i j} \\
& \frac{\mathrm{d} v_{i}}{\mathrm{~d} t}=-\sum_{j=1}^{N} m_{j}\left(\frac{p_{i}}{\rho_{i}^{2}}+\frac{p_{j}}{\rho_{j}^{2}}\right) \cdot \nabla_{i} W_{i j}+\sum_{j=1}^{N} \frac{4 m_{j}\left(\mu_{i}+\mu_{j}\right) x_{i j} \cdot \nabla_{i} W_{i j}}{\left(\rho_{i}+\rho_{j}\right)^{2}\left(x_{i j}^{2}+0.01 h^{2}\right)} v_{i j}+g .
\end{aligned}
$$

\subsection{Modeling incompressible flow with artificial compressibility}

In the SPH method, an artificial compressibility technique is usually used to model the incompressible flow as a slightly compressible flow. The artificial compressibility considers that every theoretically incompressible fluid is actually compressible. Therefore, it is feasible to use a quasi-incompressible equation of state to model the incompressible flow. The purpose of introducing the artificial compressibility is to produce the time derivative of pressure. In this work, the artificial equation of state is

$p=c^{2} \rho$,

where $c$ is the sound speed which is a key factor that deserves careful consideration. If the actual sound speed is employed, the real fluid is approximated as an artificial fluid, which is ideally incompressible. Monaghan argued that the relative density variation $\delta$ is 
related to the fluid bulk velocity and sound speed in the following way [41]

$\delta=\frac{\Delta \rho}{\rho_{0}}=\frac{\left|\rho-\rho_{0}\right|}{\rho_{0}}=\frac{V_{b}^{2}}{c^{2}}=M^{2}$,

where $\rho_{0}, \Delta \rho, V_{b o}$ and $M$ are the initial density, absolute density variation, fluid bulk velocity and Mach number respectively.

Morris, through considering the balance of pressure, viscous force and body force, proposed an estimate for the sound speed [42]. He argued that the square of the sound speed should be comparable with the largest value of $V_{b}^{2} / \delta, v V_{b} / \delta l$ and $F l / \delta$, i.e.

$c^{2}=\max \left(\frac{V_{b}^{2}}{\delta}, \frac{v V_{b}}{\delta l}, \frac{F l}{\delta}\right)$,

where $v(v=\mu / \rho)$ is the kinetic viscosity, $F$ is the magnitude of the external body force, and $l$ is the characteristic length scale.

\subsection{Density and kernel gradient correction}

It is known that the conventional SPH method has been hindered with low accuracy as it cannot exactly reproduce quadratic and linear functions, and even cannot exactly reproduce a constant. The accuracy of the conventional SPH method is also closely related to the distribution of particles, selection of smoothing function and the support domain (described by the smoothing length $h$ multiplied by a scalar factor). During the last decade, different approaches have been proposed to improve the particle inconsistency and hence the SPH approximation accuracy. Some of them involve reconstruction of a new smoothing function so as to satisfy the discretized consistency conditions. However, these approaches are usually not preferred for hydrodynamic simulations because the reconstructed smoothing function can be partially negative, non-symmetric, and not monotonically decreasing. Recently, one popular way is to construct improved SPH approximation schemes based on Taylor series expansion on the SPH approximation of a function and/or its derivatives. Typical examples include the corrective smoothed particle method (CSPM) by Chen et al. [43] and the finite particle method (FPM) by Liu et al. [44,45]. Both CSPM and FPM do not need to reconstruct smoothing function. It is noted that in the conventional SPH method, a field function and its derivatives are approximated separately. Instead, in CSPM, the derivatives are approximated through solving a coupled matrix equation while the field function is approximated separately. In FPM, both the field function and its derivatives are coupled together and can be approximated simultaneously through solving a general matrix equation.

Liquid sloshing is usually associated with changing and breakup of free surfaces. When wave front violently impacts onto solid walls of the container, water particles can first be splashed away from bulky fluid, and then fall onto the bulky fluid. The changing and breakup of free surfaces as well as splashing and fall of water particles lead to highly disordered particle distribution, which can seriously influence computational accuracy of SPH approximations. Hence an SPH approximation scheme, which is of higher order accuracy and is insensitive to disordered particle distribution, is necessary for modeling liquid sloshing dynamics.

In this work, we used two modified schemes for approximating density (density correction) and kernel gradient (kernel gradient correction, or KGC). As to the density correction, a re-normalization approach is used, in which the density can be approximated as

$\rho_{i}^{\text {new }}=\sum_{j=1}^{N} \rho_{j} W_{i j}^{\text {new }} \frac{m_{j}}{\rho_{j}}=\sum_{j=1}^{N} m_{j} W_{i j}^{\text {new }}$,
$W_{i j}^{\text {new }}=\frac{W_{i j}}{\sum_{j=1}^{N} W_{i j} \frac{m_{j}}{\rho_{j}}}$.

As to kernel gradient correction, from Eq. (2), it is known that the approximation accuracy of the derivatives is closely related to the accuracy of the gradient of the smoothing function (or kernel gradient). It is possible to use a corrective kernel gradient rather than the conventional kernel gradient in Eq. (2) to obtain better approximation accuracy. For example, in a two-dimensional space, based on Taylor series expansion on the SPH approximation of a function, it is possible to get the following formulation

$$
\begin{aligned}
\int_{\Omega} f\left(\boldsymbol{r}^{\prime}\right) \nabla W \mathrm{~d} \boldsymbol{r}^{\prime}= & f(\boldsymbol{r}) \int_{\Omega} f\left(\boldsymbol{r}^{\prime}\right) \nabla W \mathrm{~d} \boldsymbol{r}^{\prime}+\frac{\partial f(\boldsymbol{r})}{\partial x} \int_{\Omega}\left(x^{\prime}-x\right) \nabla W \mathrm{~d} \boldsymbol{r}^{\prime} \\
& +\frac{\partial f(\boldsymbol{r})}{\partial y} \int_{\Omega}\left(y^{\prime}-y\right) \nabla W \mathrm{~d} \boldsymbol{r}^{\prime}+O\left(h^{2}\right) .
\end{aligned}
$$

Considering the following equation,

$\langle\nabla f(\boldsymbol{r})\rangle=\int_{\Omega} f\left(\boldsymbol{r}^{\prime}\right) \nabla W \mathrm{~d} \boldsymbol{r}^{\prime}-f(\boldsymbol{r}) \int_{\Omega} \nabla W \mathrm{~d} \boldsymbol{r}^{\prime}$,

a new formulation for approximating derivatives can be obtained

$\langle\nabla f(\boldsymbol{r})\rangle=\frac{\partial f(\boldsymbol{r})}{\partial x} \int_{\Omega}\left(x^{\prime}-x\right) \nabla W \mathrm{~d} \boldsymbol{r}^{\prime}+\frac{\partial f(\boldsymbol{r})}{\partial y} \int_{\Omega}\left(y^{\prime}-y\right) \nabla W \mathrm{~d} \boldsymbol{r}^{\prime}+O\left(h^{2}\right)$.

It is can be further written as follows in terms of particle approximation

$$
\begin{aligned}
\left\langle\nabla f\left(\boldsymbol{r}_{i}\right)\right\rangle= & \frac{\partial f\left(\boldsymbol{r}_{i}\right)}{\partial x_{i}} \underbrace{\sum_{j}\left(x_{j}-x_{i}\right) \nabla_{i} W_{i j} V_{j}}_{X} \\
& +\frac{\partial f\left(\boldsymbol{r}_{i}\right)}{\partial y_{i}} \underbrace{\sum_{j}\left(y_{j}-y_{i}\right) \nabla_{i} W_{i j} V_{j}}_{Y}+O\left(h^{2}\right),
\end{aligned}
$$

where $V_{j}\left(=m_{j} / \rho_{j}\right)$ is the volume of particle $j$. From Eq. (15), it can be concluded that if $X=\left(\begin{array}{l}1 \\ 0\end{array}\right)$ and $Y=\left(\begin{array}{l}0 \\ 1\end{array}\right)$, the SPH particle approximation scheme for a gradient (expressed in Eq. (15)) is of second order accuracy. However, for general cases (e.g., irregular particle distribution, variable smoothing length, and/or truncated boundary areas), these two requirements cannot be satisfied, and therefore the accuracy of Eq. (15) can be seriously reduced. It is possible to restore the accuracy for general cases with the following correction on the kernel gradient,

$$
\begin{aligned}
& \nabla_{i}^{\mathrm{new}} W_{i j}=L\left(\boldsymbol{r}_{i}\right) \nabla_{i} W_{i j}, \\
& L\left(\boldsymbol{r}_{i}\right)=\left(\sum_{j}\left(\begin{array}{cc}
x_{j i} \frac{\partial W_{i j}}{\partial x_{i}} & y_{j i} \frac{\partial W_{i j}}{\partial x_{i}} \\
x_{j i} \frac{\partial W_{i j}}{\partial y_{i}} & y_{j i} \frac{\partial W_{i j}}{\partial y_{i}}
\end{array}\right) V_{j}\right)^{-1},
\end{aligned}
$$

where $x_{j i}=x_{j}-x_{i}, y_{j i}=y_{j}-y_{i}$. If replacing $\nabla_{i} W_{i j}$ on the RHS of Eq. (15) with $\nabla_{i}^{\text {new }} W_{i j}$, it is easy to verify that $X=\left(\begin{array}{l}1 \\ 0\end{array}\right)$ and $Y=\left(\begin{array}{l}0 \\ 1\end{array}\right)$. Therefore, for general cases with irregular particle distribution, variable smoothing length, and/or truncated boundary areas, the SPH particle approximation scheme for a gradient based on kernel gradient correction is of second order accuracy. It is noted that for both density correction, and gradient correction, since only kernel and its gradient are corrected, there is no need to significantly change the structure of SPH computer programs and procedure of SPH simulations. It is therefore convenient to implement SPH equations of motion. 


\subsection{RANS turbulence model}

Liquid sloshing usually involves strong turbulence and vortex, and a turbulence model is necessary to be incorporated into SPH equations of motion. Early SPH works did not include turbulence models. Recently a number of researchers gradually incorporated turbulence models into SPH equations of motion [46,47]. It is demonstrated that turbulence modeling is important for problems or areas with strong turbulence and vortex.

In this work, the Reynolds Averaged method is used to deal with Navier-Stokes equations. In the equations, a new variable can be obtained by time averaging,

$\bar{\phi}=\lim \frac{1}{t} \int_{t_{0}}^{t_{0}+t} \phi \mathrm{d} t$.

The transient volume $\phi$ is divided into two parts, the average volume $\bar{\phi}$ and the pulse volume $\phi^{\prime}\left(\phi=\bar{\phi}+\phi^{\prime}\right)$. Therefore, the Reynolds Average equations can be described as follows

$\frac{\mathrm{d} \rho}{\mathrm{d} t}=-\rho \nabla \cdot \boldsymbol{V}$

$\frac{\mathrm{d} \boldsymbol{V}}{\mathrm{d} t}=-\frac{1}{\rho} \nabla P+\frac{\mu}{\rho} \nabla^{2} \boldsymbol{V}+\mathbf{g}+\frac{1}{\rho} \nabla(\rho \boldsymbol{R})$,

where $V$ and $P$ are the new averaged volume of the original velocity and pressure, and $R$ is the Reynolds strain tensor. As Eq. (20) is no longer closed, an eddy viscosity assumption is used [48].

$R_{i j}=2 \boldsymbol{v}_{t} S_{i j}-\frac{2}{3} k \delta_{i j}$

$\boldsymbol{v}_{t}=l^{2} \sqrt{2 S_{i j} S_{i j}}$

$S_{i j}=\frac{1}{2}\left(\frac{\partial \boldsymbol{V}_{j}}{\partial x_{i}}+\frac{\partial \boldsymbol{V}_{i}}{\partial x_{j}}\right)$,

where $v_{t}$ is the turbulence eddy viscosity, $S_{i j}$ is the average strain rate tensor, and $k$ is the kinetic energy, $l$ is the maxing length, $l=C_{s} h, C_{s}$ is the Smagorinsky constant. In this paper, $C_{s}=0.12$. Finally, the tensor term in particle approximation form can be obtained as

$\frac{1}{\rho} \nabla(\rho \boldsymbol{R})=\sum_{j=1}^{N}\left(\frac{R_{i}}{\rho_{i}}+\frac{R_{j}}{\rho_{j}}\right) \nabla_{i} W_{i j}$.

\subsection{A coupled dynamic solid boundary treatment (SBT) algorithm}

One major challenge in SPH simulation is the solid boundary treatment (SBT) and implementation of the solid boundary conditions. In SPH, solid boundary conditions are not able to be directly and rigorously implemented as in the grid-based numerical models. Since its invention, the treatment of solid boundary has been a numerical focus, which has been influencing the accuracy of $\mathrm{SPH}$, and hindering its further development and application in engineering and sciences.

In this work, we presented a coupled dynamic solid boundary treatment algorithm. In this coupled SBT algorithm, two types of virtual particles, repulsive particles and ghost particles (as shown in Fig. 1), are used to represent the solid boundary. The repulsive particles produce a suitable repulsive force to the approaching fluid particles near the boundary, and they are located right on the solid boundary. Ghost particles are located outside the solid boundary area. It should be noted that, in conventional SBT algorithms, ghost particles are generated from mirroring or reflecting fluid particles onto solid boundary areas, and need to adapt with the fluid particles at each time step. In contrast, this new SBT algo-

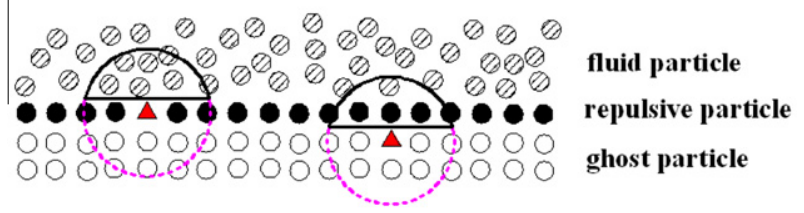

Fig. 1. Illustration of the coupled dynamic SBT algorithm.

rithm can generates ghost particles in a regular or irregular distribution at the first time step, while ghost particle positions do not need to change during following steps.

The new SBT algorithm consists of a new repulsive force for repulsive particles, and a new numerical scheme to approximate the information of the virtual particles. The new repulsive force is a distance-dependent repulsive force with finite magnitude on fluid particles approaching solid boundaries

$\boldsymbol{F}_{i j}=0.01 c^{2} \cdot \chi \cdot f(\eta) \cdot \frac{\boldsymbol{x}_{i j}}{r_{i j}^{2}}$,

$\eta=r_{i j} /\left(0.75 h_{i j}\right)$

$\chi=1-\frac{r_{i j}}{\Delta d}, \quad 0<r_{i j}<\Delta d$,

$f(\eta)= \begin{cases}2 / 3 & 0<\eta \leqslant 2 / 3 \\ \left(2 \eta-1.5 \eta^{2}\right) & 2 / 3<\eta \leqslant 1, \\ 0.5(2-\eta)^{2} & 1<\eta<2, \\ 0 & \text { otherwise }\end{cases}$

where $r$ is the distance between two particles, and $\Delta d$ is the initial distance of two adjacent particles. The improved soft repulsive force can prevent unphysical particle penetration without obvious pressure disturbances as previous boundary treatment algorithms with highly repulsive forces (such as the Lennard-Jones molecular force [20]).

In this coupled dynamic SBT algorithm, field variable of the virtual particles (both repulsive particles and ghost particles, see Fig. 1) can be dynamically evolved and obtained from SPH approximation of neighbor fluid particles within the support domain. The information of repulsive particles only comes from fluid particles, and the information of ghost particles comes from both fluid and repulsive particles. It is known that the support domains of the fluid particles intersect the solid boundary with insufficient neighbor particles. Therefore, to restore consistency, SPH particle approximation schemes with higher order accuracy (e.g. Shepard filter method, moving least square (MLS) method, CSPM or FPM) can be used in the coupled dynamic SBT algorithm for approximating both the fluid and virtual particles.

Take non-slip boundary condition for example, variables of the boundary particles (both repulsive and ghost particles) can be obtained as follows:

$\rho_{i}=\sum_{j=1}^{N} \rho_{j} W_{i j}^{\text {new }} \frac{m_{j}}{\rho_{j}}=\sum_{j=1}^{N} m_{j} W_{i j}^{\text {new }}$,

$\boldsymbol{v}_{i}=-\sum_{j}^{N} \boldsymbol{v}_{i} W_{i j}^{\text {new }} \frac{m_{j}}{\rho_{j}}$.

It has been demonstrated that improved SPH particle approximations with higher order accuracy can lead to much better results than conventional SPH particle approximation schemes [49]. It should be noted that existing modifications in SPH particle approximations with Shepard filter or moving least square have only been 


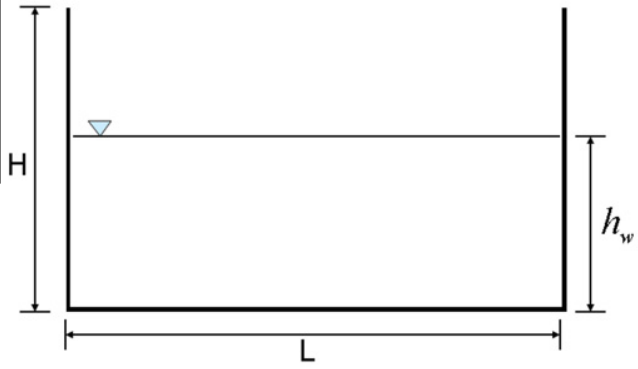

Fig. 2. Illustration of the liquid sloshing system.

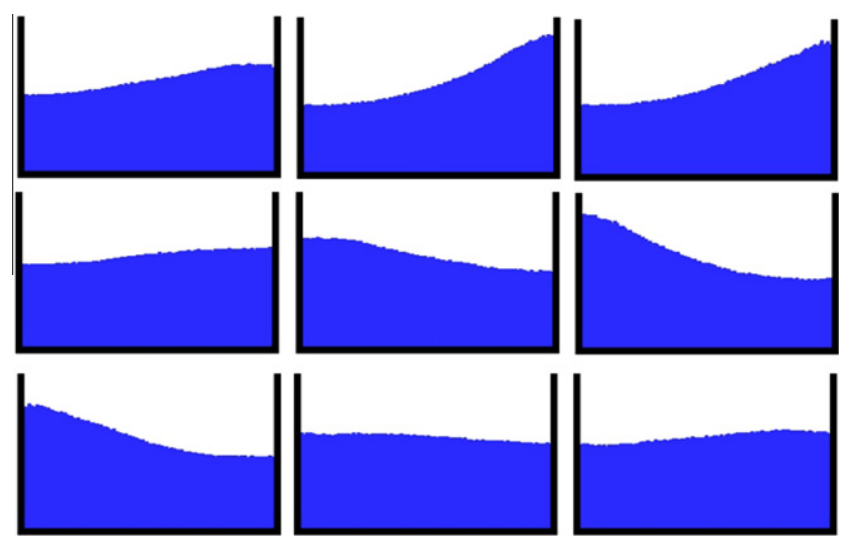

Fig. 3. Particle distributions at 20, 20.2, 20.4, 20.6, 20.8, 21, 21.2, 21.4 and $21.5 \mathrm{~s}$.

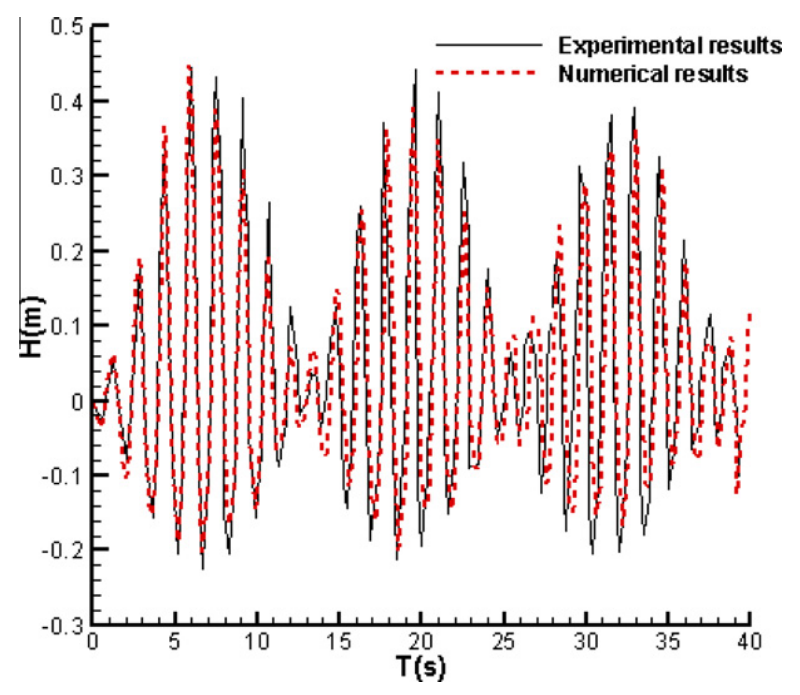

Fig. 4. Wave heights obtained by SPH simulation and experiment.

used for approximating information of fluid particles. This is the first time to extend the idea of using higher order accuracy schemes to approximate information of virtual particles in treating solid boundaries. And therefore the improved interpolation scheme serves to improve the computational accuracy for calculating the information of virtual particles.

\section{Numerical examples}

In this section, three numerical examples related to liquid sloshing shall be simulated. The first example involves liquid slosh-
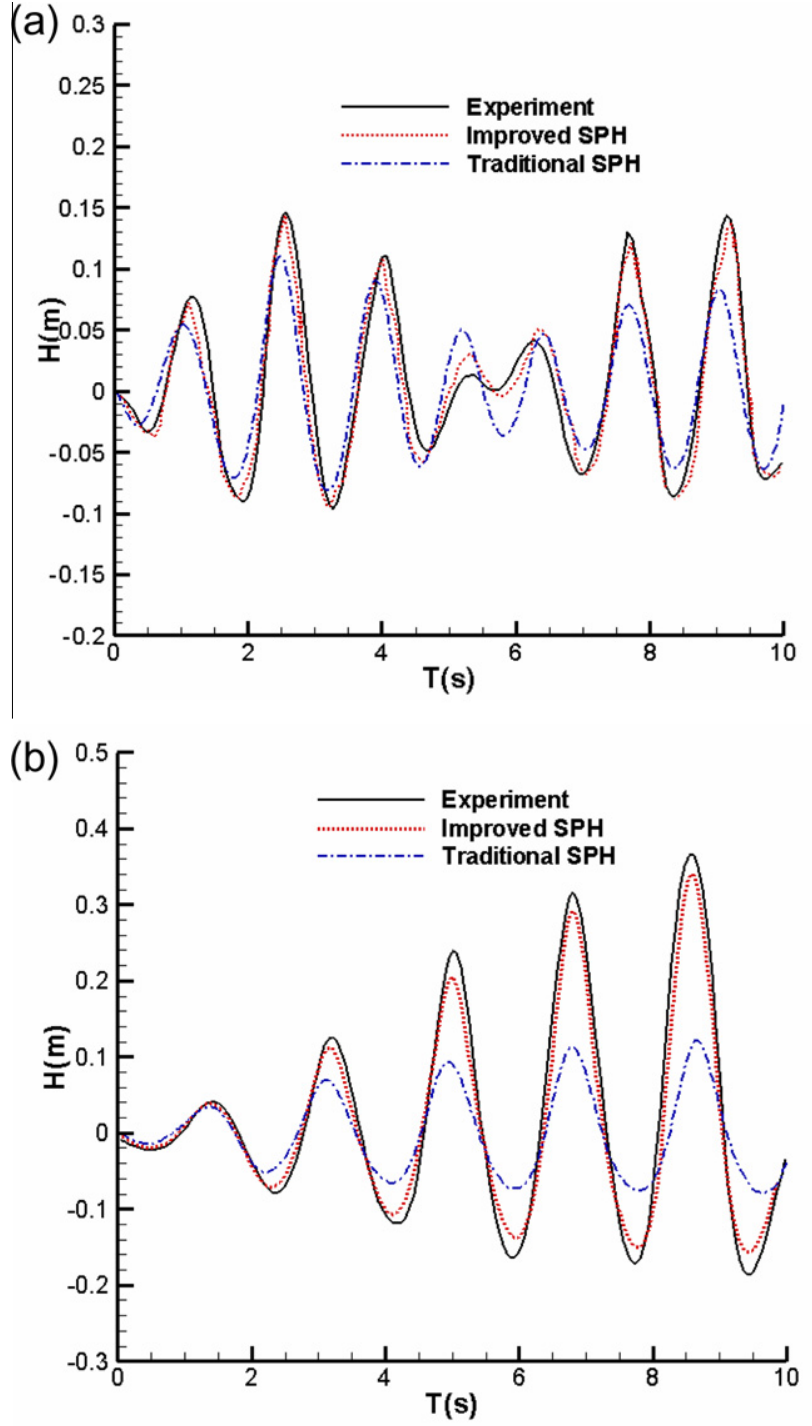

Fig. 5. Wave heights for two scenarios with $h_{w}=0.6 \mathrm{~m}, T=1.3 \mathrm{~s}(\mathrm{a})$, and $h_{w}=0.5 \mathrm{~m}$, $T=1.875 \mathrm{~s}$ (b).

ing in a rectangular tank with a horizontal excitation and the wave height near the left wall is tracked. In the second one, a middle baffle is placed in a water tank, and factors influencing liquid sloshing are investigated. The third example is related to the pitch motion of water in the rectangular tank. SPH results are comparatively investigated with experimental observations and results from other sources.

\subsection{Liquid sloshing under horizontal excitation}

In this case, the liquid motion in a rectangular tank under a periodic horizontal excitation is simulated. Fig. 2 shows the geometry of the liquid sloshing system, which is similar to what Faltinsen et al. provided [10], i.e., $L=1.73 \mathrm{~m}, h_{w}=0.6 \mathrm{~m}$, and $H=1.15 \mathrm{~m}$. For liquid sloshing with small amplitudes, there is no water impact on the top of the water tank. Under external excitation, the water tank moves according to $S=A \cos (2 \pi t / T)$, where $S$ is the location of the tank, $A$ and $T$ are amplitude and period of the external excitation, and are taken as $0.032 \mathrm{~m}$ and $1.5 \mathrm{~s}$. The corresponding Reynold's number $\left(R e=V_{C} \bullet L / v\right.$, where $V_{C}$ is the characteristic velocity from the movement of the tank, and $v$ is the kinetic 

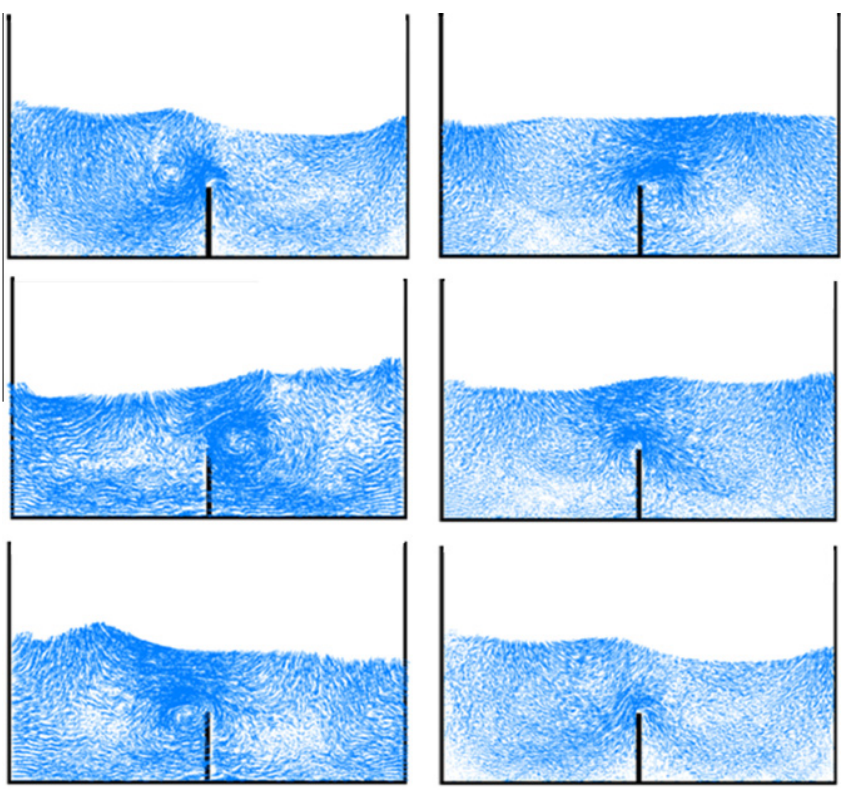

Fig. 6. Velocity vector distributions at $6.0,6.3,6.6,6.9,7.2$ and $7.5 \mathrm{~s}$ while the height of the middle baffle $(d)$ is $0.3 \mathrm{~m}$.
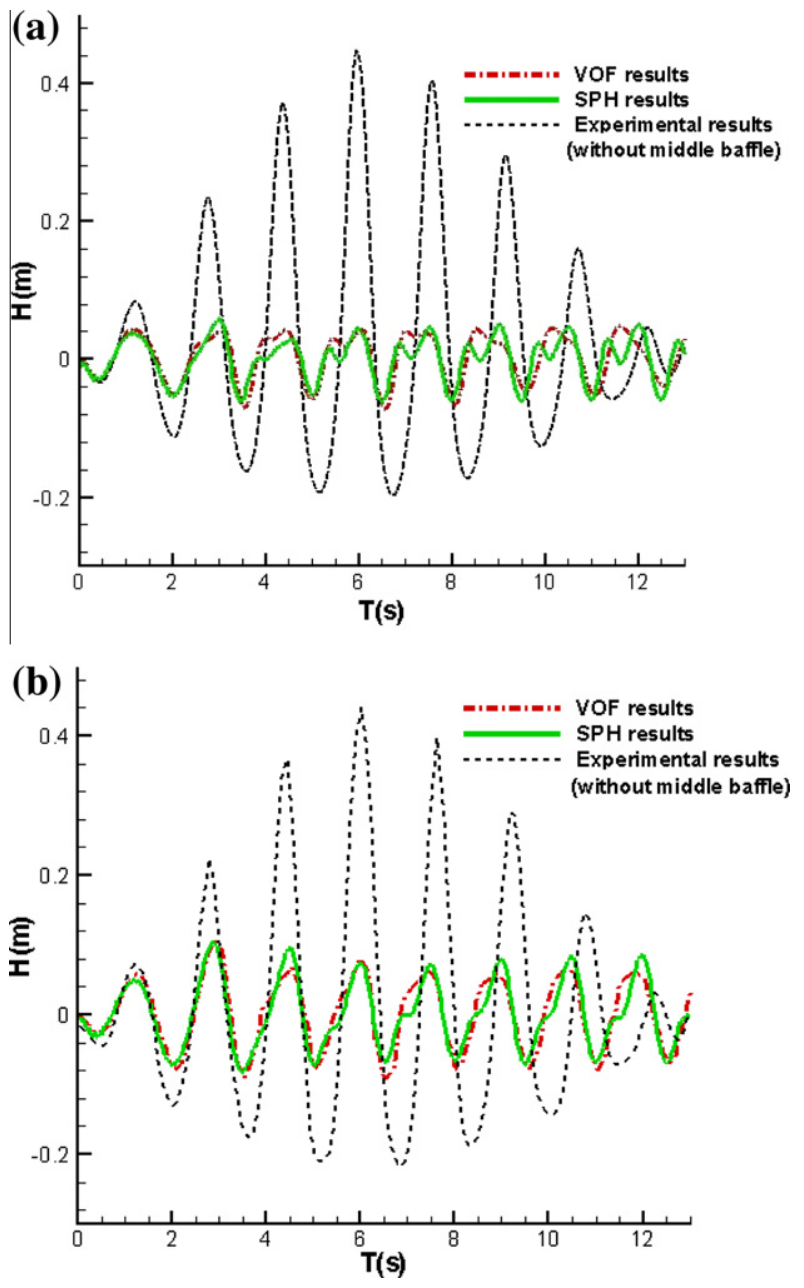

Fig. 7. Wave heights with different heights of baffle: (a) $d=0.3 \mathrm{~m}$ and (b) $d=0.4 \mathrm{~m}$.

viscosity of water) is approximately $0.23 \times 10^{6}$. A probe is placed on the initial free surface, and is $0.05 \mathrm{~m}$ away from the left wall.

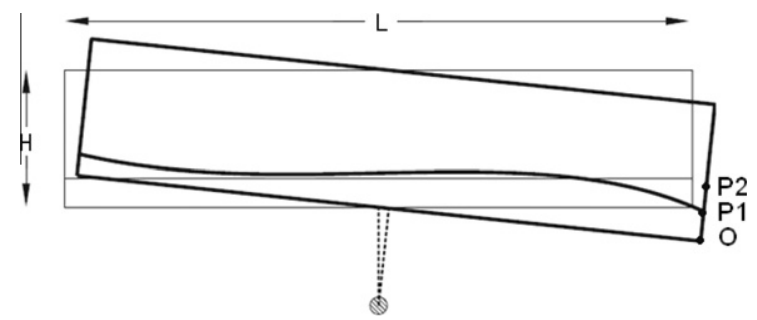

Fig. 8. Liquid sloshing due to the pitch motion of a rectangular tank.

In the SPH simulation, the time step is taken as $5 \times 10^{-6} \mathrm{~s}$, and around 40000 particles are used. The reciprocal movement of the water tank drives the contained water to and fro in the tank. Fig. 3 shows the flow pattern of sloshing at nine typical time instants within one period. As the water tank begins to move rightward, the contained water also starts to move rightward. Due to the blocking effect of the right wall, water particles will gradually aggregate and tends to run up along the right wall until a maximum water height is reached. After then water particles near the top right corner will then gradually fall down, move leftward, gradually aggregate and tends to run up along the left wall, and finally reach a maximum water height followed by new period.

Fig. 4 shows the wave heights obtained by SPH simulation and experiment [10] at the probe point in a $40 \mathrm{~s}$ time course. The SPH results agree well with experimental observations, both in pattern, period and amplitude. There are some discrepancies in wave trough areas, which may be due to the sparser particle distribution when water reaches the lowest point.

Changing initial water height and period of the external excitation can lead to different liquid sloshing phenomena. Fig. 5 shows the wave heights for two scenarios with $h_{w}=0.6 \mathrm{~m}, T=1.3 \mathrm{~s}$, and $h_{w}=0.5 \mathrm{~m}, T=1.875 \mathrm{~s}$. Again for both scenarios, the obtained SPH results are comparable with experimental observations. Numerical results obtained using traditional SPH model (with simple repulsive boundary treatment, without density and kernel gradient correction) are also provided for these two scenarios. It is seen that the improved SPH model can get more agreeable results with experimental observations.

\subsection{Liquid sloshing with a middle baffle}

This example involves liquid sloshing in a rectangular tank with a middle baffle. The baffle can changes the behavior of liquid sloshing, reduces the wave heights, and therefore help to suppress the impact load of water onto the tank. The geometry and external excitation are the same as the above case (i.e., $h_{w}=0.032 \mathrm{~m}$ and $T=1.5 \mathrm{~s}$ ) except for the use of the baffle. In this simulation, the time step is taken $5 \times 10^{-6} \mathrm{~s}$, the Reynold's number is approximately $R e \approx 0.23 \times 10^{6}$, and around 24000 particles are used.

Fig. 6 shows the velocity vector distribution at 6.0, 6.3, 6.6, 6.9, 7.2 , and $7.5 \mathrm{~s}$ while the height of the middle baffle $(d)$ is $0.3 \mathrm{~m}$. It is observed that a number of vortices have been reciprocally generated and disappeared. There is a large eddy around the top of the baffle, and it evolves quickly with the movement of the water particles. This reveals that the improved SPH method with the RANS turbulence model can effectively describe the inherent turbulence physics. These vortices and eddies complicate the liquid sloshing behavior, dissipate energy, and help to alleviate impact and vibration effects due to liquid sloshing.

To investigate the influence of different heights of middle baffle, a probe is also placed on the initial free surface, and is $0.05 \mathrm{~m}$ away from the left wall to track the wave height. Fig. 7 shows the wave heights with different height of baffle ( $a, d=0.3 \mathrm{~m}$; $\mathrm{b}, d=0.4 \mathrm{~m}$ ) obtained using SPH and VOF simulations. The comparative work 

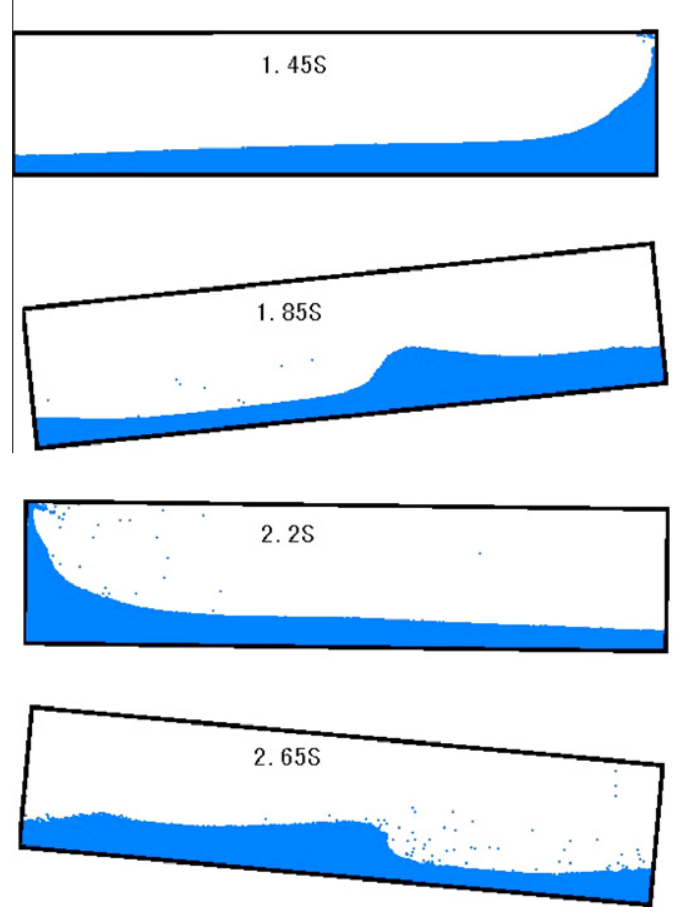

Fig. 9. Particle distributions at 1.45, 1.65, 1.85, 1.98, 2.2, 2.45, 2.65 and $2.90 \mathrm{~s}$.
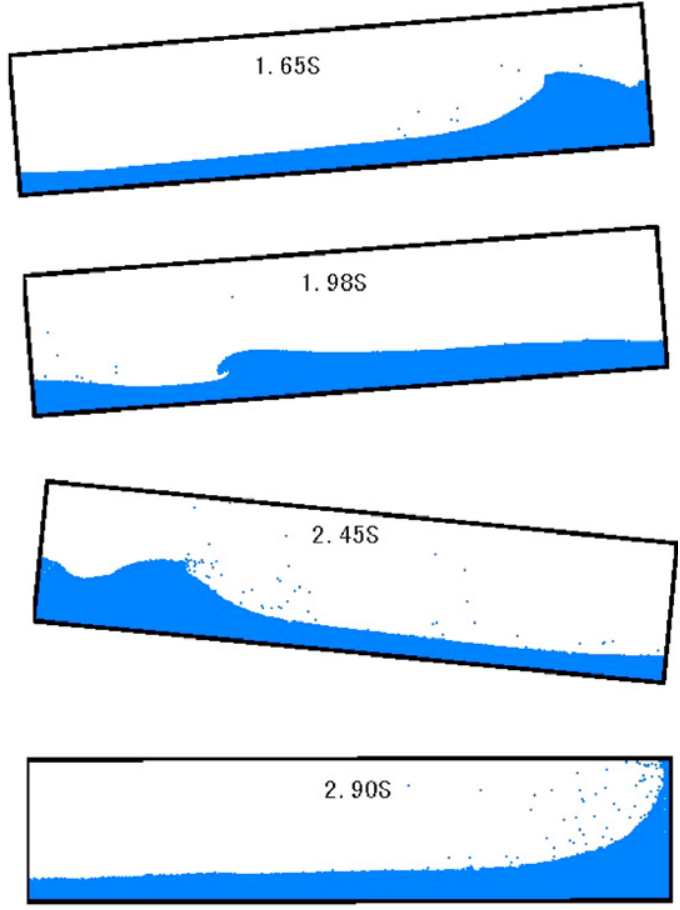

(a)

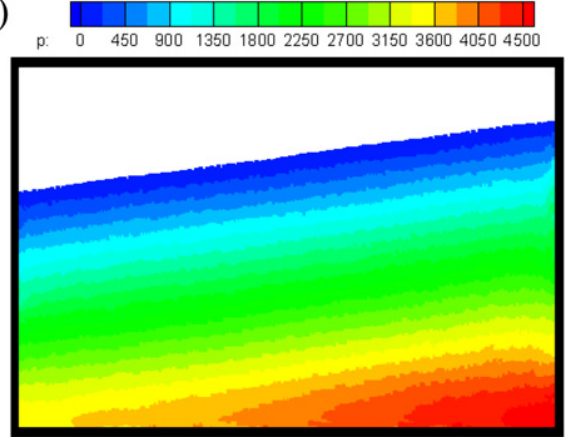

(b)

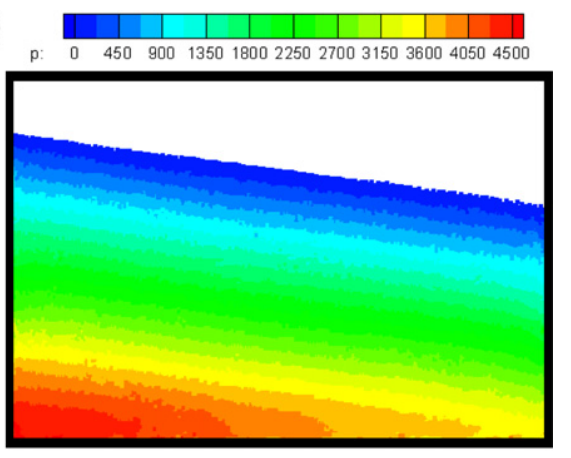

Fig. 10. Pressure field with $\theta_{0}=8^{\circ}$ at $7.2 \mathrm{~s}$ (a) and $8.8 \mathrm{~s}(\mathrm{~b})$.

was done using commercial software, Fluent, with the VOF model to track free surface, while in the VOF model, the geometric reconstruction scheme is used to calculate the face fluxes [50]. In the VOF simulation, about 28000 mesh cells (24 000 particles are used in the corresponding SPH model) are used, and the time step $0.001 \mathrm{~s}$. Experimental observations of liquid sloshing in the same rectangular tank without middle baffle are also provided. It is clear that the obtained SPH results agree well with the results obtained using VOF to track free surfaces. Compared with experimental data without middle baffle, it is seen that using a middle baffle greatly reduces the wave height. Also changing the height of the baffle can lead to different behavior of liquid sloshing including the period and amplitude of the wave heights. When $d=0.3 \mathrm{~m}$, the maximum wave height is around $0.1 \mathrm{~m}$. Increasing the baffle height to $d=0.4 \mathrm{~m}$ can reduced the maximum wave height to around $0.05 \mathrm{~m}$.

It should be noted that though SPH and VOF results are close, there are some discrepancies. For both cases with the middle baffle, SPH results clearly demonstrate double wave troughs in a period, a main wave trough with an additional one. The double wave trough is closely related to the middle baffle. With a middle baffle, water wave in the liquid tank can be influenced, with a number of major or small vertices as shown in Fig. 6. The middle baffle can even lead to second water waves on both sides. The accumulation of the major wave and the second wave produces a water wave with double wave trough. While in VOF simulation, there are no (Fig. 7a) or only ambiguous (Fig. 7b) double wave troughs in a period. This shows that the improved SPH method is more effective in resolving inherent complex flow patterns due to liquid sloshing with separated baffles.

\subsection{Liquid sloshing due to the pitch motion of a rectangular tank}

In this case, the tank is allowed to rotate around the transverse axis, and hence the pitch motion of a rectangular tank is studied. The external excitation can be described as $\theta=\theta_{0} \sin \left(\omega_{r} t+\xi_{0}\right)$, where $\theta_{0}$ is the angular displacement, $\omega_{r}$ is the circular frequency of the pitch motion, and $\xi_{0}$ is the initial phase. Fig. 8 shows an illustration of the problem geometry.

We firstly used following parameters to model the liquid sloshing due to the pitch motion of a rectangular tank, i.e., $\theta_{0}=6^{\circ}$, $\omega_{r}=4.34 \mathrm{rad} / \mathrm{s}, \quad \xi_{0}=0, L=0.64 \mathrm{~m}, H=0.14 \mathrm{~m}$. The water depth 
$\left(h_{w}\right)$ is $0.03 \mathrm{~m}$, and the center of rotation is $0.1 \mathrm{~m}$ below the baseline. In this case, about 24000 particles were used, and the Reynold's number is $0.16 \times 10^{6}$ approximately.

Fig. 9 shows the particle distribution due to the pitch motion of a rectangular tank. The improved SPH method can effectively capture the flow dynamics associated with changing and breaking free surfaces. At around $1.45 \mathrm{~s}$, water particles run up along the right wall and then impact onto the right and top side of the tank, with some particles splashed away from the bulky water. After then, water particles fall downwards, and propagate leftwards, with some splashed water particles falling onto the bulky water. With the leftward propagating movement of water, a number of surge fronts appear, depending on the geometry and angular velocity of the water tank as well as water height. Later, water particles aggregate and run up along the left wall and then impact onto the left and top side of the tank, also with some particles splashed away from the bulky water. As such, a half period ends, followed by symmetric flow pattern during the next half period.

We also studied another liquid sloshing problem with the same setup as Akyildiz and Erdem [51], in which $H=0.62 \mathrm{~m}, L=0.92 \mathrm{~m}$, $\theta=\theta_{0} \cos \left(\omega_{r} t+\xi_{0}\right), \omega_{r}=2 \mathrm{rad} / \mathrm{s}, \xi_{0}=\pi / 2$, the filled depth is $75 \%$, and the model rotates around the tank center. on the right wall, two probes are set to track the pressure values, as shown in Fig. $8, O P 1=0.06 \mathrm{~m}, P 1 P 2=0.11 \mathrm{~m}$. In this case, about 20000 particles were used, and the Reynold's number is $0.13 \times 10^{6}$ approximately. Two external excitations with different angular displacement $\left(\theta_{0}=4^{\circ}\right.$ or $\left.8^{\circ}\right)$ are simulated.

Fig. 10 shows the obtained pressure field with $\theta_{0}=8^{\circ}$ at $7.2 \mathrm{~s}(\mathrm{a})$ when the peak pressure values can be observed at the two probe points $\mathrm{P} 1$ and $\mathrm{P} 2$, and at 8.8 (b) s, when the minimum pressure value can be observed. It is noted that different from traditional SPH models, which usually lead to rough and oscillated pressure distribution, the improved SPH method can produce smooth pressure distribution with clear pressure layers. Even near the solid boundary area, there are no obvious pressure oscillations.

Figs. 11 and 12 show the pressure values at two probes, P1 and $\mathrm{P} 2$, with two different angular displacement, $\theta_{0}=4^{\circ}$ and $\theta_{0}=8^{\circ}$.
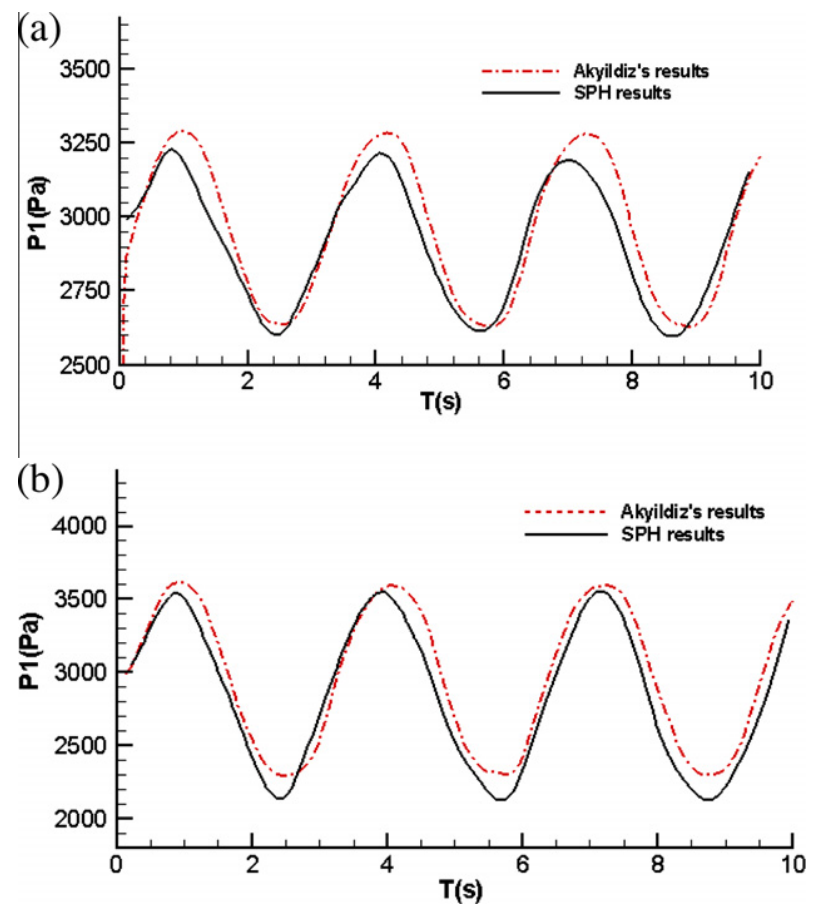

Fig. 11. Pressure values at probe P1 with different angular displacement: (a) $\theta_{0}=4^{\circ}$ and (b) $\theta_{0}=8^{\circ}$
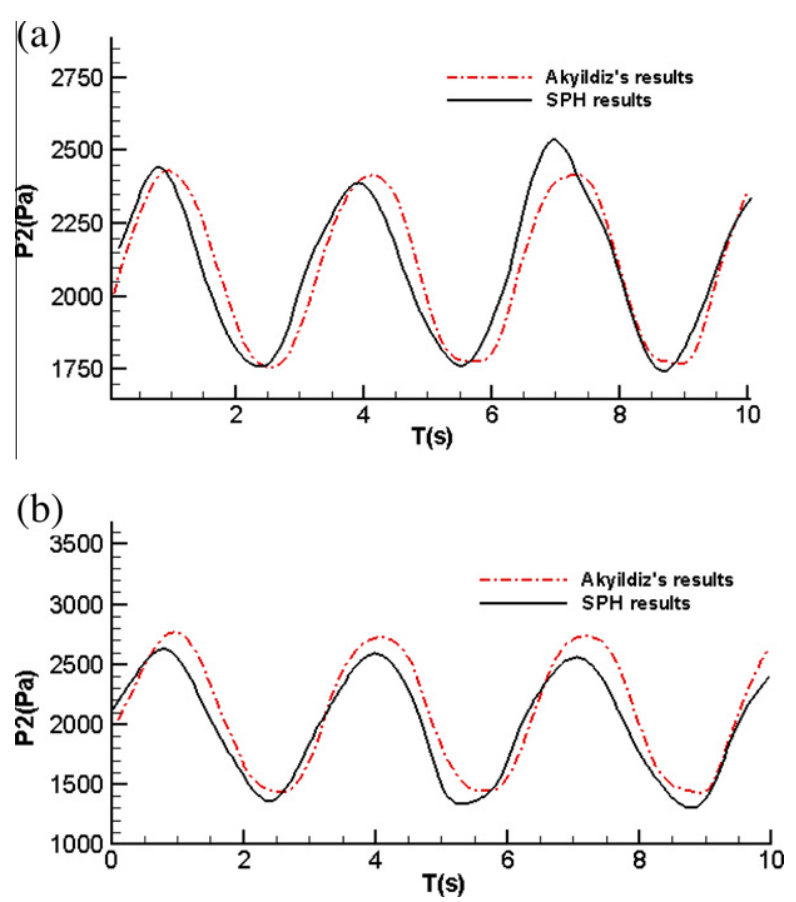

Fig. 12. Pressure values at probe $P 2$ with different angular displacement: (a) $\theta_{0}=4^{\circ}$ and (b) $\theta_{0}=8^{\circ}$.

It is seen that with the movement of the water tank, the measured pressure values rise and fall periodically. The obtained SPH results agree in general with the results provided by Akyildiz and Erdem [51], who used a VOF model to track free surfaces. This reveals that the improved SPH method with kernel gradient and density correction and coupled dynamic solid boundary treatment algorithm are effective in resolving pressure field, and in treating solid boundaries.

\section{Conclusions}

This paper presented an improved SPH method for modeling viscous incompressible liquid sloshing dynamics. The improved SPH method includes corrections on density and kernel gradient approximation, enhancement in solid boundary treatment, and incorporation of turbulence model. Density correction and kernel gradient correction can help to improve computational accuracy for modeling viscous impressible flows with changing and breaking free surfaces. The structure of SPH computer program and procedure of SPH simulation do not need significant change with density and kernel gradient correction. The coupled dynamic solid boundary treatment algorithm uses two types of virtual particles to describe the solid boundary, either fixed in computational domain or moving with the rigid object. The kernel-like, soft repulsive force between approaching fluid and solid particles, on one hand, can effectively prevent particles from unphysical penetration, and on the other hand, can avoid unwanted numerical oscillations in solid boundary area. The coupled dynamic SBT algorithm also uses approximation schemes with higher order accuracy for estimating field functions of virtual solid particles, and therefore it can enhance computational accuracy in boundary areas. The incorporated RANS turbulence model is able to model flows with strong turbulence and vortex,

To demonstrate the effectiveness of the improved SPH method for liquid sloshing dynamics, three numerical examples are provided. These examples are associated with different external excitations and different structures. It is seen that the SPH method can 
well capture physics related to changing and breaking surfaces. The SPH method with RANS turbulence model also can effectively resolve vortices and eddies, which can dissipate energy, and alleviate impact and vibration on solid walls of water tank. The obtained numerical results including flow pattern, wave height, pressure field, and pressure load on solid walls are agreeable with experimental observations or results from other sources.

\section{Acknowledgements}

This work has been supported by the National Natural Science Foundation of China (11172306) and National Defense Innovation Funds of the Chinese Academy of Sciences (Y175031XML).

\section{References}

[1] Ibrahim RA. Liquid sloshing dynamics: theory and applications. Cambridge Univ Pr; 2005.

[2] Ibrahim RA, Pilipchuk VN, Ikeda T. Recent advances in liquid sloshing dynamics. Appl Mech Rev. 2001;54:133-99.

[3] Faltinsen OM, Timokha AN. Sloshing: Cambridge Univ Pr; 2009.

[4] Cariou A, Casella G. Liquid sloshing in ship tanks: a comparative study of numerical simulation. Mar Struc. 1999;12:183-98.

[5] Chen BF, Nokes R. Time-independent finite difference analysis of fully nonlinear and viscous fluid sloshing in a rectangular tank. J Comput Phys. 2005;209:47-81.

[6] Chen BF. Viscous fluid in tank under coupled surge, heave, and pitch motions. J Waterw Port Coast Ocean Eng. 2005;131:239.

[7] Wu GX, Ma QW. Eatock Taylor R. Numerical simulation of sloshing waves in a 3D tank based on a finite element method. Appl Ocean Res. 1998;20:337-55.

[8] Mitra S, Upadhyay PP, Sinhamahapatra KP. Slosh dynamics of inviscid fluids in two-dimensional tanks of various geometry using finite element method. Int J Numer Method Fluid. 2008;56:1625-51.

[9] Faltinsen OM. A numerical nonlinear method of sloshing in tanks with twodimensional flow. J Ship Res 1978:22.

[10] Faltinsen OM, Rognebakke OF, Lukovsky IA, Timokha AN. Multidimensional modal analysis of nonlinear sloshing in a rectangular tank with finite water depth. J Fluid Mech. 2000;407:201-34.

[11] Faltinsen OM, Timokha AN. An adaptive multimodal approach to nonlinear sloshing in a rectangular tank. J Fluid Mech. 2001;432:167-200.

[12] Faltinsen OM, Timokha AN. A multimodal method for liquid sloshing in a twodimensional circular tank. J Fluid Mech. 2010;665:457-79.

[13] Liu DM, Lin PZ. A numerical study of three-dimensional liquid sloshing in tanks. J Comput Phys. 2008;227:3921-39.

[14] Veldman AEP, Gerrits J, Luppes R, Helder JA, Vreeburg JPB. The numerical simulation of liquid sloshing on board spacecraft. J Comput Phys. 2007;224:82-99.

[15] Fang ZY, Duan MY, Zhu RQ. Numerical simulation of liquid sloshing in a liquid tank based on Level-set method. J Ship Mech. 2007;11:62.

[16] Pan XJ, Zhang HX, Lu YT. Numerical simulation of viscous liquid sloshing by moving-particle semi-implicit method. J Mar Sci Appl. 2008;7:184-9.

[17] Koshizuka S, Nobe A, Oka Y. Numerical analysis of breaking waves using the moving particle semi-implicit method. Int J Numer Method Fluid. 1998;26:751-69.

[18] Gingold RA, Monaghan JJ. Smoothed particle hydrodynamics-theory and application to non-spherical stars. Mon Notic Roy Astron Soc. 1977; $181: 375-89$

[19] Lucy LB. A numerical approach to the testing of the fission hypothesis. Astron J. 1977;82:1013-24.

[20] Liu GR, Liu MB. Smoothed particle hydrodynamics: a meshfree particle method. Singapore: World Scientific; 2003.

[21] Liu MB, Liu GR, Lam KY. Constructing smoothing functions in smoothed particle hydrodynamics with applications. J Comput Appl Math. 2003;155:263-84.

[22] Hu XY, Adams NA. An incompressible multi-phase SPH method. J Comput Phys. 2007;227:264-78.
[23] Tartakovsky AM, Meakin P. A smoothed particle hydrodynamics model for miscible flow in three-dimensional fractures and the two-dimensional Rayleigh Taylor instability. J Comput Phys. 2005;207:610-24.

[24] Tartakovsky AM, Tartakovsky DM, Scheibe TD, Meakin P. Hybrid simulations of reaction-diffusion systems in porous media. Siam J Sci Comput. 2007;30:2799-816.

[25] Libersky LD, Petschek AG, Carney TC, Hipp JR, Allahdadi FA. High strain Lagrangian hydrodynamics: a three-dimensional SPH code for dynamic material response. J Comput Phys. 1993;109:67-75.

[26] Randles PW, Libersky LD. Smoothed particle hydrodynamics: Some recent improvements and applications. Comput Method Appl Mech Eng. 1996;139:375-408.

[27] Johnson GR, Beissel SR. Normalized smoothing functions for SPH impact computations. Int J Numer Method Eng. 1996;39:2725-41.

[28] Liu MB, Liu GR, Lam KY. Adaptive smoothed particle hydrodynamics for high strain hydrodynamics with material strength. Shock Waves. 2006;15:21-9.

[29] Swegle JW, Attaway SW. On the feasibility of using Smoothed Particle Hydrodynamics for underwater explosion calculations. Computation Mech. 1995;17:151-68.

[30] Liu MB, Liu GR, Lam KY, Zong Z. Smoothed particle hydrodynamics for numerical simulation of underwater explosion. Computation Mech. 2003;30:106-18.

[31] Liu MB, Liu GR, Zong Z, Lam KY. Computer simulation of high explosive explosion using smoothed particle hydrodynamics methodology. Comput Fluids. 2003;32:305-22.

[32] Liu MB, Liu GR. Smoothed Particle Hydrodynamics (SPH): an Overview and Recent Developments. Arch Comput Method Eng. 2010;17:25-76.

[33] Cleary PW, Prakash M, Ha J, Stokes N, Scott C. Smooth particle hydrodynamics: status and future potential. Prog Comput Fluid Dyn. 2007;7:70-90.

[34] Monaghan JJ. Smoothed particle hydrodynamics. Rep Progr Phys. 2005;68:1703-59.

[35] Iglesias AS, Rojas LP, Rodriguez RZ. Simulation of anti-roll tanks and sloshing type problems with smoothed particle hydrodynamics. Ocean Eng. 2004;31:1169-92.

[36] Rhee SH, Engineer L. Unstructured grid based Reynolds-averaged NavierStokes method for liquid tank sloshing. J Fluid Eng. 2005;127:572.

[37] Souto-Iglesias A, Delorme L, Perez-Rojas L, Abril-Perez S. Liquid moment amplitude assessment in sloshing type problems with smooth particle hydrodynamics. Ocean Eng. 2006;33:1462-84.

[38] Anghileri M, Castelletti LML, Tirelli M. Fluid structure interaction of water filled tanks during the impact with the ground. Int J Impact Eng. 2005;31:235-54.

[39] Delorme L, Iglesias AS, Perez SA. Sloshing loads simulation in LNG tankers with $\mathrm{SPH}$. International Conference on Computational Methods in Marine Engineering. Barcelona, 2005.

[40] Kalitzin G, Medic G, Iaccarino G, Durbin P. Near-wall behavior of RANS turbulence models and implications for wall functions. J Comput Phys. 2005;204:265-91.

[41] Monaghan JJ. Simulating free surface flows with SPH. J Comput Phys 1994;110:399.

[42] Morris JP, Fox PJ, Zhu Y. Modeling low Reynolds number incompressible flows using SPH. J Comput Phys. 1997;136:214-26.

[43] Chen JK, Beraun JE. A generalized smoothed particle hydrodynamics method for nonlinear dynamic problems. Comput Method Appl Mech Eng. 2000;190:225-39.

[44] Liu MB, Liu GR. Restoring particle consistency in smoothed particle hydrodynamics. Appl Numer Math. 2006;56:19-36.

[45] Liu MB, Xie WP, Liu GR. Modeling incompressible flows using a finite particle method. Appl Math Model. 2005;29:1252-70.

[46] Shao SD, Hitoshi G. Turbulence particle models for tracking free surfaces. J Hydraul Res. 2005;43:276.

[47] Shao SD, Incompressible SPH. Simulation of wave breaking and overtopping with turbulence modelling. Int J Numer Method Fluid. 2006;50:597-621.

[48] Lo E, Shao SD. Simulation of near-shore solitary wave mechanics by an incompressible SPH method. Appl Ocean Res. 2002;24:275-86.

[49] Colagrossi A, Landrini M. Numerical simulation of interfacial flows by smoothed particle hydrodynamics. J Comput Phys. 2003;191:448-75.

[50] Fluent Inc. User guide of Fluent Software. 2005.

[51] Akyildiz H. Erdem Unal N. Sloshing in a three-dimensional rectangular tank: Numerical simulation and experimental validation. Ocean Eng. 2006;33:2135-49. 